REVISIÓN

\title{
Asociación entre leche adulterada con melamina y aparición de litiasis renal en población infantil: una revisión de literatura de los casos acaecidos en la República Popular China
}

\author{
Jasmina Bordón-González a , José Roig-Beltrán a y Rocío Ortiz-Moncada a,b,* \\ ${ }^{a}$ Departamento de Enfermería Comunitaria, Medicina Preventiva y Salud Pública e Historia de la Ciencia, \\ Universidad de Alicante, Alicante, España \\ 'Observatorio de Políticas Públicas y Salud y Grupo de Investigación Salud Pública, Universidad de Alicante, España
}

Recibido el 27 de septiembre de 2011; aceptado el 14 de marzo de 2012

\author{
PALABRAS CLAVE \\ Sustitutos de la leche; \\ Melamina; \\ Adulteración de \\ alimentos; \\ Nefrolitiasis; \\ Cálculo renal; \\ Niños; \\ Epidemiología
}

\begin{abstract}
Resumen
Introducción: La melamina es una sustancia química usada en la producción de resinas. Cuando se añade a la leche, aumenta la concentración de nitrógeno, lo que indica un falso incremento de proteína y puede producir problemas renales en algunos subgrupos de población. El objetivo del trabajo es documentar la posible relación entre la leche industrializada adulterada con melamina y la aparición de litiasis renal en menores de 12 años mediante la revisión de casos acaecidos en la República Popular China.

Material y métodos: Se realizó una revisión bibliográfica de artículos publicados entre 2005 y 2010 y un análisis descriptivo.

Resultados: Se obtuvieron 20 artículos, 11 cumplían criterios de inclusión: nueve estudios transversales, uno de seguimiento, uno de casos y controles. Cuatro estudios calculan odds ratio (OR) en sus análisis, para evaluar el riesgo relativo de padecer cálculos renales en población infantil expuesta a la fórmula de leche adulterada con melamina (FLM). En el estudio de casos y controles, la población infantil expuesta a la FLM tenía 5,17 veces más probabilidad de padecer cálculos renales que los expuestos a la fórmula de leche sin melamina (intervalo de confianza del 95\%, 3,28-8,14; $p<0,001$ ).

Conclusiones: La adulteración de la leche con melamina es un problema de salud pública evitable que indica que hay que establecer más medidas de control y de aplicación de la legislación alimentaria, tanto por parte de la industria alimentaria como por las autoridades sanitarias y alimentarias.

(c) 2011 Asociación Española de Dietistas-Nutricionistas. Publicado por Elsevier España, S.L. Todos los derechos reservados.
\end{abstract}

${ }^{*}$ Autor para correspondencia.

Correo electrónico: rocio.ortiz@ua.es (R. Ortiz-Moncada). 


\section{KEYWORDS}

Milk substitutes;

Melamine;

Food adulteration;

Nephrolithiasis;

Kidney calculi;

Infant;

Epidemiology
Relationship Between Milk Adulterated With Melamine and the Appearance of Renal Stones in a Childhood Population: A Review of the Literature of the Cases That Occurred in the People's Republic of China

\begin{abstract}
Introduction: Melamine is a chemical substance used in the production of resins. When melamine is added to milk, nitrogen concentration increases, which suggest an increase the amount of false proteins and consequently causing kidney diseases in some population subgroups. The objective of this study is to document the possible connection in between adulterated industrial milk with melamine and the appearance of kidney stones in children under 12 years old by reviewing cases occurred in the People's Republic of China.

Material and methods: A literature review was carried out on articles published on dated between 2005-2010, and an extensive analysis was made on the results found. Results: A total of 20 articles were reviewed, of which 11 met the criteria for inclusion, There were 9 cross-sectional studies, one follow-up study and a case-control study. Four studies calculated odds ratio $(\mathrm{OR})$ in their analysis to evaluate the relative risk of having stones in those children exposed to melamine formula. In the case-control study, children exposed to melamine formula were 5.17 times as likely to have kidney stones as children exposed to a non-melamine formula (95\% confidence interval, 3.28-8.14; $P<.001$ ).

Conclusions: Melamine adulteration in milk is a preventable public health issue which suggests the establishing of greater control measures and the implementation of food regulations by the food industry and Health and Food Institutions.

(c) 2011 Asociación Española de Dietistas-Nutricionistas. Published by Elsevier España, S.L. All rights reserved.
\end{abstract}

\section{Introducción}

La melamina, definida por el sistema IUPAC (International Union of Pure and Applied Chemistry) como 2,4,6-triamino1,3,5-triazina, es una sustancia química usada industrialmente en la producción de resinas para la elaboración de productos laminados, colas, adhesivos y plásticos ${ }^{1}$. En la industria láctea, especialmente en la referida al sector infantil, se ha usado la melamina como una forma de adulterar los sustitutivos de la leche para falsear la cantidad de proteína presente y pasar los controles de calidad ${ }^{2}$. La melamina aumenta la concentración de nitrógeno, lo que indica un falso aumento de la proteína del producto. Un caso claro de adulteración generalizada de la leche con melamina por la industria láctea se dio en la República Popular China; sin embargo, también se ha usado en otros países, incluso en la Unión Europea ${ }^{3}$.

Dispensar alimentos inocuos es una de las condiciones esenciales y uno de los objetivos principales de las agencias nacionales e internacionales de seguridad alimentaria y nutrición. Es decir, que todas las personas en el mundo tengan acceso físico y económico a alimentos inocuos y nutritivos con el fin de satisfacer sus necesidades y preferencias alimentarias, para que así puedan llevar una vida activa y saludable ${ }^{4-6}$. Sin embargo, se reconocen problemas en la higiene y la inocuidad de los alimentos, lo que conlleva un grave problema de inseguridad alimentaria de poblaciones específicas ${ }^{4,5}$.

Las causas de los problemas que puedan ocurrir en la seguridad alimentaria pueden ser sucesos accidentales o comportamientos negligentes. Generalmente, son los comportamientos deliberados, cuyo móvil es el enriquecimiento, el origen de los fraudes alimentarios. En estos casos, la calidad o las condiciones del producto que se comercializará se ven afectadas, y se hace necesario implantar políticas de seguridad y control. Es decir, es preciso crear directrices y programas para controlar la disponibilidad, el acceso, la distribución y la utilización de los alimentos y ofrecer criterios claros a los consumidores al elegir los alimentos que consumen ${ }^{6,7}$. Con estas medidas, se intenta prevenir las prácticas fraudulentas, la adulteración de alimentos y cualquier otra práctica que pueda inducir al engaño, no sólo por medio de controles físicos, químicos y biológicos de materias primas, procesos y productos ${ }^{7}$, sino también controlando el etiquetado, la publicidad y la presentación de alimentos para no inducir a error a los consumidores ${ }^{6}$.

El fraude de adulteración de la proteína de la leche constituyó un antecedente destacado en la salud pública en el mundo. Las primeras noticias sobre la adulteración de la leche con melamina datan del 11 de septiembre de 2008 en China. Hay estudios que muestran que aproximadamente el $14 \%$ de las fórmulas infantiles fabricadas ese año contenían melamina ${ }^{8}$. Uno de los probables efectos del consumo de sustitutivos de la leche adulterados fue la aparición de urolitiasis en población infantil. El objetivo del presente estudio es documentar, a través de una revisión bibliográfica de casos acaecidos en la República Popular China publicados entre mayo de 2005 y mayo de 2010, la posible relación entre la leche industrializada adulterada con melamina y la aparición de litiasis renal en menores de 12 años. 
Asociación entre leche adulterada con melamina y aparición de litiasis renal en población infantil: una revisión de literatura de los casos acaecidos en la República Popular China

\section{Material y métodos}

Estudio descriptivo de artículos científicos publicados entre 2005 y 2010 recuperados mediante explotación y revisión bibliográfica de bases de datos electrónicas especializadas. Las bases de datos consultadas fueron: PubMed, Scopus y TripDatabase. La estrategia o ecuación de búsqueda se construyó a partir de los descriptores incluidos en el Medical Subject Headings (MeSH) de Index Medicus/MEDLINE: "Milk" AND "Melamine" AND "Kidney Calculi" AND "Infant" AND “Child, Preschool” AND “Child”.

Los criterios de inclusión definidos fueron: artículos originales (texto completo), idiomas (español e inglés), publicados en cualquier país entre mayo de 2005 y mayo de 2010, población infantil entre 0 y 12 años afectada por ingerir fórmula de leche adulterada con melamina. Se excluyeron los editoriales, las cartas al editor, los artículos de revisión bibliográfica y los artículos empíricos sin datos de la población sujeta a estudio.

Variables de estudio: a) país y año de estudio, país donde se realizó el estudio y año en el cual se efectuó; $b$ ) diseño del estudio, se refiere al tipo y diseño de estudio que aparece en la parte metodológica de artículo: transversal, de seguimiento, descriptivos, analíticos entre otros; c) población y muestra, los determinados en el artículo; $d$ ) variables de estudio y recogida de información, definidas como las variables que los autores consignaron en el manuscrito; $e$ ) resultados del estudio, se define como la medida de frecuencia (prevalencia) de la enfermedad consignada en los artículos, y $f$ ) existe asociación, se refiere a que los autores en cada estudio aceptan la relación del desarrollo de cálculos renales con el consumo de leche adulterada con melamina, en los estudios que especifican la asociación.

Para recoger la información de las variables de estudio se construyó un protocolo (tabla 1). Los artículos que cumplían con los criterios de inclusión se recuperaron en texto completo vía internet o por compra. Se realizó un análisis descriptivo de los resultados.

\section{Resultados}

Se encontró un total de 20 artículos, de los que 11 cumplían todos los criterios de inclusión $2,8-17$. Se identificaron 9 artículos en inglés $2,8,10-16$ y 2 estudios escritos en chino, con resumen en inglés ${ }^{9,17}$.

En la tabla 1 se muestra el resumen de las características y variables de estudio de los artículos incluidos en el análisis descriptivo de la revisión bibliográfica. Los estudios, pese a su año de publicación, se realizaron entre 2008 y 2009 en China, años en que la noticia se dio a conocer. Básicamente se trata de estudios transversales $(n=9)^{2,8-11,13}$ y 2 de seguimiento ${ }^{12,17}$. La ecografía fue el método diagnóstico de litiasis renal en todos los estudios. Para la recogida de la información se utilizaron cuestionarios para declarar diagnósticos médicos de litiasis renal, entre otras variables de estudio. En otros estudios, la información se obtuvo a través de la historia clínica y de la historia alimen$\operatorname{taria}^{8,11,15,16}$.

Las variables de estudio se definen de acuerdo con el objetivo del estudio. En aquellos cuyo objetivo es abordar la fiabilidad y la precisión de métodos diagnósticos ${ }^{13,15}$ y de pronóstico en el tratamiento ${ }^{14,16,17}$, las variables de exposición se relacionan con la ingesta de la fórmula de leche adulterada con melamina (FLM), la concentración de melamina en la leche, el tiempo y la duración de la exposición a la FLM. La variable dependiente se relaciona con la presencia de cálculos renales $(C R)$ determinados por síntomas clínicos y método diagnóstico. En los otros estudios, las variables se complementan con otras pruebas analíticas y bioquímicas, examen clínico, entre otros ${ }^{2,8-12}$.

Para presentar los resultados de población y muestra, prevalencia de litiasis renal (CR asociados a melamina [CRAM]) y la variable de si existe probable asociación, presentadas en la tabla 1 , se reagruparon los estudios en cuatro subtablas: $A$. Estudios descriptivos cuyo objetivo es el diagnóstico; $B$. Estudios transversales de prevalencia; $C$. Estudios transversales descriptivos y analíticos, y $D$. Estudios longitudinales analíticos.

En la tabla $1 \mathrm{~A}$ se relacionan dos estudios descriptivos cuyo objetivo es el diagnóstico ${ }^{16}$ y afinar el tratamiento con mayor eficacia ${ }^{16,17}$, partiendo de población infantil con cálculos renales expuestos al consumo de FLM. Aunque los autores no concluyen una posible asociación, puesto que no es su objetivo de estudio, el hecho de que su población sean casos se relaciona con el desarrollo de la litiasis renal con la exposición a la leche adulterada.

La tabla $1 \mathrm{~B}$ se refiere a estudios descriptivos transversales $(n=5)$ que parten de población expuesta a FLM y la aparición de cálculos renales, cuya prevalencia varía del 0,03 al 3,1\%2,8,13-15. Lam et $\mathrm{al}^{2}$ (2008) y Zhu et $\mathrm{al}^{8}$ (2009), muestran que la prevalencia y la gravedad de las complicaciones renales en los afectados puede explicarse por la diferencia en los niveles altos de exposición a melamina. Los otros tres estudios atañen a objetivos diagnósticos como, por ejemplo, mostrar que la ecografía es un método preciso y fiable ${ }^{13}$, conocer las características ecográficas de la litiasis urinaria causada por FLM ${ }^{15}$ y las características del tratamiento ${ }^{14}$. Al tratarse de estudios descriptivos, los autores concluyen que existe relación entre cálculos renales en los niños y la exposición a la leche adulterada con melamina.

La tabla $1 \mathrm{C}$ relaciona los estudios transversales descriptivos y analíticos 9 -11 con prevalencias mayores que las descritas en la tabla 3. Hu et $\mathrm{al}^{9}$ (2008) y Zhang et $\mathrm{al}^{10}$ (2009) muestran que la prevalencia de CR es mayor cuando los pacientes han sido alimentados con fórmulas de leche adulterada con altas concentraciones de melamina. La regresión logística mostró asociación estadísticamente significativa entre el desarrollo de CR y el consumo prolongado de fórmula de leche adulterada con alto contenido de melamina (FLAM) y, además con el consumo mínimo de agua $(p<0,05)$, aunque fueron determinantes independientes con una variación de $18,2 \%{ }^{10}$. Por otro lado, los resultados de la regresión logística múltiple indican que la relación del sexo con la incidencia de litiasis urinaria es estadísticamente significativa $(p<0,05)$. Las niñas presentan mayor incidencia $(58,7 \%)$ de $C R$ y se observa menor riesgo en los niños (odds ratio [OR] = 0,587; intervalo de confianza del $95 \%$ [IC95\%], 0,359-0,959). Los autores concluyen que la incidencia de litiasis urinaria en niños de 0-3 años de edad es relativamente alta9. Guan et $\mathrm{al}^{11}$ (2009) corroboran los hallazgos de que la exposición a la fórmula adulterada con 


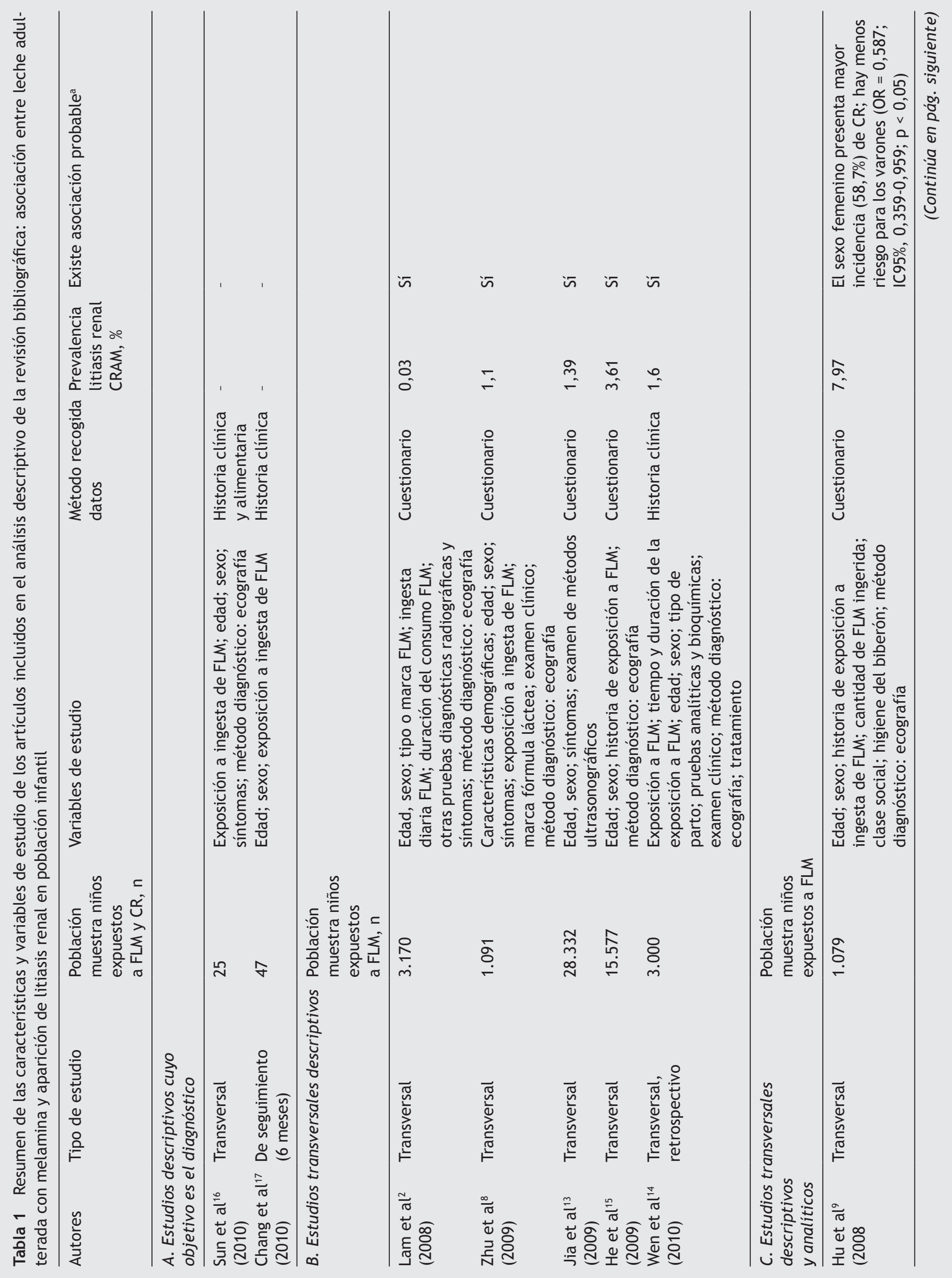




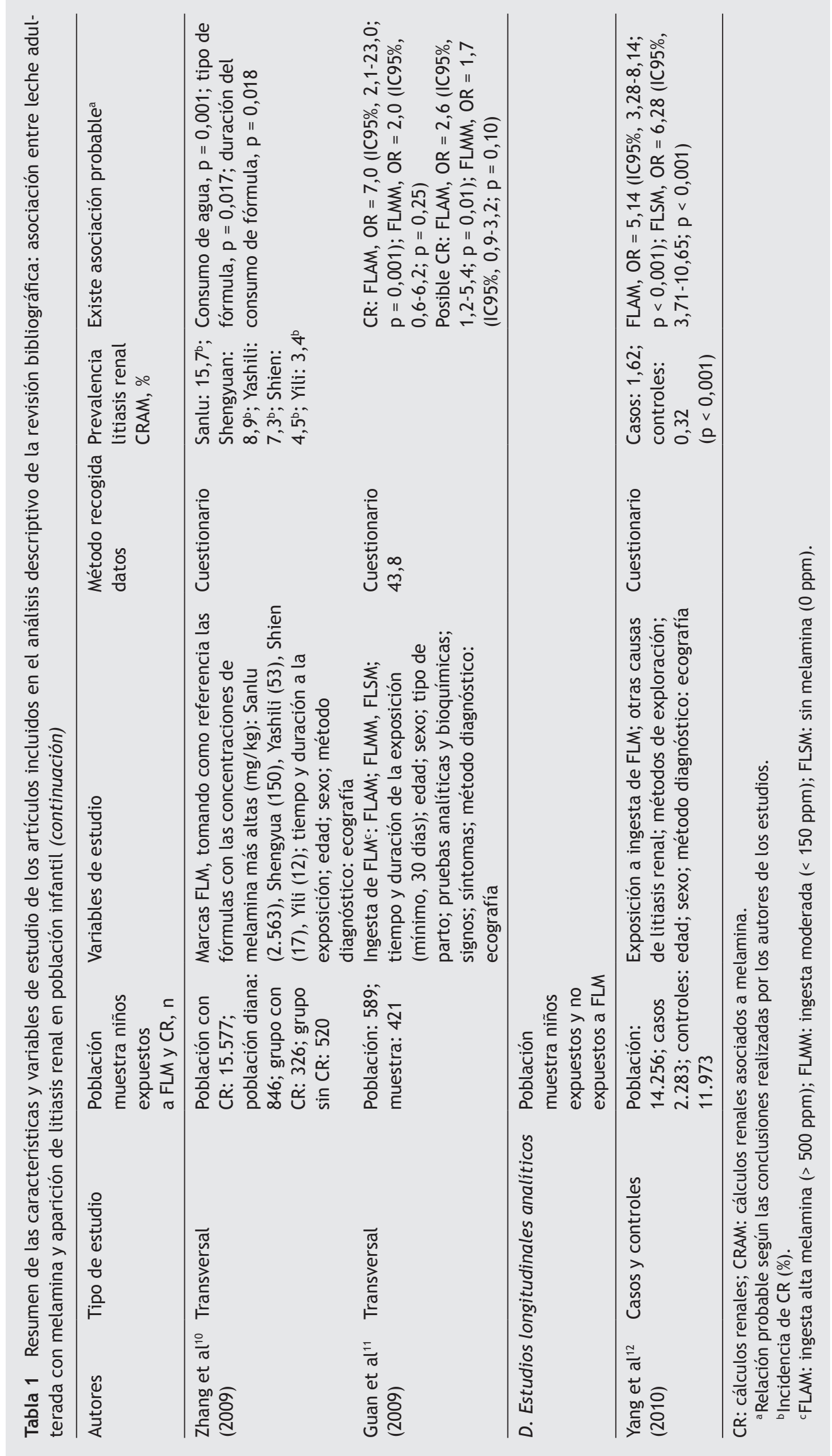


melamina se asoció con cálculos urinarios y con la prematuridad. La población expuesta a FLAM presenta 7,0 veces más probabilidad de tener CR que los no expuestos a FLM. Los recién nacidos prematuros eran 4,5 veces más propensos a tener cálculos que los nacidos a término.

Por último, en la tabla 1D se relaciona el único estudio longitudinal, de casos y controles, encontrado en la revisión bibliográfica. Yang et $\mathrm{al}^{12}$ (2010) muestran que la exposición a la ingesta de la FLM se asocia significativamente con cálculos urinarios.

La prevalencia de litiasis renal en la población infantil expuesta a la FLM $(1,62 \%)$ fue significativamente más alta que la población expuesta a fórmula sin contenido de melamina $(0,32 \%)(p<0,001)$. Además, los niños expuestos a fórmulas con altas concentraciones de melamina eran 5,14 veces más propensos a desarrollar cálculos renales que las personas expuestas a fórmulas sin melamina (IC95\%, 3,28$8,14 ; p<0,001)$. También evidencian que los niños menores de 3 años expuestos a FLAM eran 6,28 veces más propensos a tener cálculos renales que los expuestos a fórmulas sin melamina (IC95\%, 3,71-10,65; p < 0,001). No hubo diferencias estadísticamente significativas entre los CR y la exposición a las fórmulas con melamina y las fórmulas sin melamina con la edad, el sexo y la ubicación del cálculo $(p>0,05)$.

\section{Discusión}

Los resultados de la revisión bibliográfica muestran que los estudios utilizan diseños epidemiológicos con suficiente poder explicativo para establecer relaciones causales. Se cuenta con un estudio de casos y controles ${ }^{12}$ y tres estudios transversales ${ }^{9-11}$, que analizan OR y corroboran la hipótesis de que la incidencia de CR es mayor en los pacientes que consumen FLAM frente a los que consumen fórmulas de leche sin melamina $(O R=5,14 ; \text { IC95\%, 3,28-8,14; } p<0,001)^{12}$. También se encuentra relación con el tiempo de exposición a esta ${ }^{10-12}$, la ingesta de agua9, ${ }^{9}$, la edad ${ }^{8,12,13}$ y el sexo $0^{9,13,14}$, entre otros factores.

La Agencia de Alimentos y Medicamentos de Estados Unidos considera que el nivel máximo de melamina permisible para el consumo humano es $0,63 \mathrm{mg} / \mathrm{kg} / \mathrm{día}^{2}$. Concentraciones superiores a esta cifra son nocivas para la salud e incrementan el riesgo de padecer nefrolitiasis e incluso insuficiencia renal aguda ${ }^{2}$. Guan et $\mathrm{al}^{11}$ concluyen que una fórmula infantil con una concentración de melamina > 500 ppm tiende a aumentar el riesgo de formación de cálculos en el sistema urinario de bebés y lactantes debido a la baja solubilidad de la melamina ${ }^{8}$.

De igual modo, los resultados muestran que el tiempo de exposición a la sustancia se asocia a un mayor riesgo de aparición de la enfermedad en cuestión; la probabilidad de tener cálculos renales es aproximadamente de $5,17^{12}$ a $7^{11}$ veces mayor en los expuestos que en los no expuestos, y dicha cifra tiende a aumentar a medida que la edad del afectado es menor ${ }^{11,12}$. La mayor prevalencia de CR se encontró en la población masculina, con una razón niños:niñas de $3: 1^{14}$.

Cabe destacar que la edad de la población más afectada no supera los 24 meses $^{8,14,15}$, debido a que la leche es el alimento principal en la alimentación de los lactantes. La leche adulterada con melamina conlleva mayores concentraciones de esta en el organismo del niño y, en consecuencia, mayor probabilidad de litiasis renal, situación que se empeora por el hecho de que al lactante le será más difícil la expulsión de los cálculos renales en comparación con los adultos ${ }^{8}$.

Los estudios evidencian que la ecografía es la mejor prueba diagnóstica para confirmar los cálculos renales en el sistema urinario causados por la melamina en combinación con ácido úrico ${ }^{13,16}$. De hecho, estos cálculos difieren de los producidos por oxalato de calcio en que son radiolúcidos, por lo que no se muestran en exámenes radiográficos ${ }^{8,15}$. Además, los cálculos causados por melamina se pueden observar en las ecografías como estructuras claras, sin sombras, contrariamente a lo que se observa en las ecografías de CR causados por acumulación de calcio ${ }^{13}$.

Es complejo conocer la magnitud del problema en la salud pública. De hecho, algunos estudios muestran que con un tratamiento adecuado, de hidratación y alcalinización, la mayoría de los casos no resultan tener complicaciones, de modo que se puede prevenir así la aparición de insuficiencia renal aguda en los menores. Incluso, un estudio muestra que los CR pueden llegar a expulsarse espontáneamente, dependiendo de su localización en el sistema urinario $^{17}$. Según la descripción del Ministerio de Salud de China, las partes más afectadas son el sistema colector y los uréteres bilaterales ${ }^{1}$. Según los estudios realizados en dicha muestra, la dificultad del diagnóstico radica principalmente en que los afectados no presentan los signos típicos de litiasis renal ${ }^{8,11,13}$. Sin embargo, Lam et $\mathrm{al}^{2}$ (2008) declaran que habría que esperar más tiempo para evaluar el impacto de dicha adulteración en la salud de la población expuesta, como especifican Wei et $\mathrm{al}^{18}$ (2011).

En China, cerca de 50.000 niños y niñas se vieron afectados, más de 100 de ellos con enfermedades graves y al menos tres casos fatales. Aunque 39.965 niños se recuperaron en corto tiempo, según información oficial facilitada por el Ministerio de Salud de China a los ciudadanos, en octubre de 2008 todavía 5.824 bebés seguían hospitalizados, de los que 6 estaban en graves condiciones ${ }^{8}$. La situación fue declarada un problema internacional de salud pública.

Aunque los 11 artículos muestran que la melamina es causa probable de nefrolitiasis en menores de 12 años, los resultados presentan limitaciones. A modo de ejemplo, dado el impacto mediático que tuvo conocerse la adulteración de la leche, al declararla los padres podrían haber sobrestimado la ingesta de melamina de los menores. Además, la concentración de melamina en las fórmulas infantiles se estimó teniendo en cuenta la información ofrecida por las marcas productoras de dichas leches, con lo que podría estar subestimada la concentración de melamina presente en las fórmulas. La Administración Estatal de Supervisión de la Calidad y de Inspección y Cuarentena de la República Popular China declara que 22 grandes marcas son las más afectadas por la adulteración. Por otro lado, los estudios se realizaron en distintas regiones del país, observándose claras diferencias en las concentraciones de melamina encontradas en las fórmulas infantiles adultera$\operatorname{das}^{2} \mathrm{y}$, por lo tanto, apreciándose grandes diferencias en los resultados de los estudios obtenidos de la búsqueda bibliográfica. 
Asociación entre leche adulterada con melamina y aparición de litiasis renal en población infantil: una revisión de literatura de los casos acaecidos en la República Popular China

La adulteración de los productos alimenticios ha tenido una doble cara, una desde las políticas públicas y otra muy diferente desde el punto de vista comercial y económico. Desde las políticas, el impacto para la salud de las poblaciones ha llevado a una mayor regulación y vigilancia de reglamentos y normas. Sin embargo, desde la rentabilidad económica, los fraudes alimentarios siguen existiendo, a pesar de conocerse el impacto negativo en la población ${ }^{6}$. La leche es un claro ejemplo de producto sujeto de adulteraciones por ser un alimento de consumo masivo y porque se puede adicionarle materias primas más económicas y de mayor disponibilidad comercial.

En alusión a las negligencias alimentarias, otro claro ejemplo de impacto negativo en la salud pública fue el caso del síndrome del aceite tóxico (Spanish toxic oil syndrome [STOS]) ocurrido en 1981 en España. El STOS causó aproximadamente 1.600 muertes y 25.000 afectados por ingerir aceite de colza ${ }^{19}$. El aceite de colza desnaturalizado con anilina al $2 \%^{20}$, apto para el uso industrial pero no para el consumo humano ${ }^{21}$, causó a los consumidores intensas mialgias, marcada eosinofilia secundaria e infiltraciones pulmonares ${ }^{22}$. Tras este hecho, se crearon medidas de regulación y control para garantizar la seguridad alimentaria y medidas de sanción por primera vez en España. Sin embargo, no se cuestionó el papel del Estado ni el de los principales implicados en la gestión de la seguridad alimentaria ${ }^{19}$. No fue hasta el año 2000, tras la aparición del primer caso de encefalopatía espongiforme bovina en España, cuando surgió una reforma significativa en la política española. Tras la crisis de las "vacas locas", se creó la Agencia Española de Seguridad Alimentaria y Nutrición (AESAN), y se adoptó un nuevo paradigma en seguridad alimentaria, cuyos nuevos objetivos se resumen en regular, controlar, sancionar y analizar riesgos alimentarios potenciales ${ }^{19}$.

En lo que respecta al ámbito internacional, la creciente globalización del comercio de los alimentos ha llevado a la creación de normas internacionales sobre inocuidad de los alimentos. En 1963, la Organización de las Naciones Unidas para la Alimentación y la Agricultura y la Organización Mundial de la Salud crearon la comisión del Codex Alimentarius $^{23}$. Debido a que los accidentes alimentarios y los brotes de enfermedades de transmisión alimentaria han repercutido en la opinión pública, se han incorporado, además de las materias de inocuidad de los alimentos, la seguridad alimentaria, la nutrición y el derecho a la alimentación de toda persona ${ }^{24}$. En 1995, el Acuerdo sobre la Aplicación de Medidas Sanitarias y Fitosanitarias y el Acuerdo sobre Obstáculos Técnicos al comercio reconocieron oficialmente que las indicaciones incluidas en el Codex Alimentarius, junto con las normas, directrices y recomendaciones internacionales, son puntos de referencia para la regulación del comercio y el derecho internacional $^{23}$.

La adaptación de un diseño basado en un marco normativo moderno armonizado con la normativa internacional, particularmente con el Codex Alimentarius, la prevención de la inocuidad en la cadena de alimentos basada en las buenas prácticas y el diseño y gestión de un sistema nacional de control de alimentos son ámbitos que habría que abarcar y asegurar para garantizar la calidad e inocuidad de productos alimenticios ${ }^{7}$.

\section{Conclusiones}

Los estudios muestran que la leche adulterada con melamina causó un brote de CR en la población infantil menor de 12 años en China, un grave problema de salud pública y de impacto mediático internacional. Este hecho es un claro ejemplo de negligencia en la aplicación rigurosa de la legislación alimentaria y de los procedimientos relativos a asegurar la calidad y la inocuidad de los alimentos destinados al consumo infantil. Un estricto control por parte de las autoridades sanitarias y alimentarias de cada país puede poner fin a tales desastres sanitarios.

\section{Conflictos de intereses}

Los autores declaran no tener ningún conflicto de intereses.

\section{Bibliografía}

1. Fink JK. Melamine resins. En: Fink JK. Reactive polymers fundamentals and applications: a concise guide to industrial polymers. New York: William Andrew Publishing; 2005. p. 299-305.

2. Lam HS, Ng PC, Chu WC, Wong W, Chan DF, Ho SS, et al. Renal screening in children after exposure to low dose melamine in Hong Kong: cross sectional study. BMJ. 2008;337:a2991.

3. Suchý P, Straková E, Herzig I, Stana J, Kalusová R, Pospíchalová $M$. Toxicological risk of melamine and cyanuric acid in food and feed. Interdiscip Toxicol. 2009;2:55-9.

4. Ortiz Moncada MR, Serra Majem L. Políticas de nutrición. En: Serra-Majem L, Aranceta J, editores. Nutrición y salud pública: métodos, bases científicas y aplicaciones. $2 .^{\text {a }}$ ed. Barcelona: Masson; 2006. p. 666-75.

5. Álvarez-Dardet Díaz C, Ortiz Moncada MR, Serra Majem L, Mata Albert $\mathrm{E}$. Peligros y riesgos sanitarios asociados a los alimentos. En: Sierra Lopez A, Saenz González MC, Fernández-Crehuet Navajas J, et al, editores. Piedrola Gil. Medicina preventiva y salud pública. 11. ${ }^{\text {a }}$ ed. Barcelona: Elsevier-Masson; 2008. p. 401-22.

6. Serra Majem L, Ribas Barba L, Ortiz Moncada MR. Políticas de nutrición y salud pública: ¿más ciencia o más política? En: Alemany $M$, Bernabeu-Mestre J. Bioética y nutrición. Salamanca: Universidad de Alicante, Editorial Agua Clara; 2010. p. 123-48.

7. Mercado C. Los ámbitos normativos, la gestión de la calidad y la inocuidad alimentaria: una visión integral. Agroalimentaria. 2007;24:119-31.

8. Zhu SL, Li JH, Chen L, Bao ZX, Zhang LJ, Li JP, et al. Conservative management of pediatric nephrolithiasis caused by melamine-contaminated milk powder. Pediatrics. 2009;123: e1099-102.

9. Hu SL, Wu L, Chen Y, Pan JH, Xu WP, Wang L, et al. Investigation on the incidence of urolithiasis in infants fed by milk power tainted with melamine in the middle area of Anhui Province. Chin J Evid-based Med. 2008;8:1039-42.

10. Zhang L, Wu LL, Wang YP, Liu AM, Zou CC, Zhao ZY. Melaminecontaminated milk products induced urinary tract calculi in children. World J Pediatr. 2009;5:31-5.

11. Guan N, Fan Q, Ding J, Zhao Y, Lu J, Ai Y, et al. Melaminecontaminated powdered formula and urolithiasis in young children. N Engl J Med. 2009;360:1067-74.

12. Yang $\mathrm{H}$, Wang Q, Luo J, Li Q, Wang L, Li CC, et al. Ultrasound of urinary system and urinary screening in 14256 asymptomatic children in China. Nephrology (Carlton). 2010;15:362-7. 
13. Jia LQ, Shen $Y$, Wang XM, He LJ, Xin Y, Hu YX. Ultrasonographic diagnosis of urinary calculus caused by melamine in children. Chin Med J (Engl). 2009;122:252-6.

14. Wen JG, Li ZZ, Zhang $H$, Wang $Y$, Zhang RF, Yang L, et al. Melamine related bilateral renal calculi in 50 children: single center experience in clinical diagnosis and treatment. J Urol. 2010;183:1533-7.

15. He Y, Jiang GP, Zhao L, Qian JJ, Yang XZ, Li XY, et al. Ultrasonographic characteristics of urolithiasis in children exposed to melamine-tainted powdered formula. World J Pediatr. 2009;5:118-21.

16. Sun $Q$, Shen $Y$, Sun $N$, Zhang GJ, Chen Z, Fan JF, et al. Diagnosis, treatment and follow-up of 25 patients with melamine-induced kidney stones complicated by acute obstructive renal failure in Beijing Children's Hospital. Eur J Pediatr. 2010;169:483-9.

17. Chang $Y M$, Tong $X M$, Wang $X L$, Zhao $Y M$. [Follow-up study on children with urinary calculi associated with exposure to melamine-contaminated formula]. Zhongguo Dang Dai Er Ke Za Zhi. 2010;12:278-9.

18. Wei Y, Liu D. Review of melamine scandal: still a long way ahead. Toxicol Ind Health. 2011 Oct 10 [Epub ahead of print].
19. Palau A. Continuidad y cambio en las políticas públicas: el caso de la política de seguridad alimentaria en España (1981-2001). Rev Esp Ciencia Política. 2009;21:47-68.

20. Almansa A, Borda IA, Abaitua MA, Banegas JR. Effects of toxic oil syndrome on the psychological conditions of the descendants of affected persons. Epidemiol Prev. 2008;32:212-7.

21. Izquierdo J. La justicia del accidente, variaciones sobre el síndrome del síndrome del aceite tóxico español. Rev Antropol Soc. 2003;12:287-320.

22. Gelpí E, De la Paz MP, Terracini B, Abaitua I, De la Cámara AG, Kilbourne EM, et al; WHO/CISAT Scientific Committee for the Toxic Oil Syndrome. Centro de Investigación para el Síndrome del Aceite Tóxico. The Spanish toxic oil syndrome 20 years after its onset: a multidisciplinary review of scientific knowledge. Environ Health Perspect. 2002;110:457-64.

23. Codexalimentarius. net [portal en internet]. Roma: Normas alimentarias FAO/OMS Online [actualizado 2011; citado 22 Jun 2011]. Disponible en: http://www.codexalimentarius.net/ web/index_es.jsp

24. Spreij M, Vapnek J. Directrices en materia de legislación alimentaria: nuevo modelo de ley de alimentos para países de tradición jurídica romano-germánica. Roma: Organización de las Naciones Unidas para la Agricultura y la Alimentación; 2006. 